\title{
Determination of the rituximab binding site to the CD20 epitope by using SPOT-synthesis and surface plasmon resonance analyses
}

\author{
Laure Bar, ${ }^{\dagger}$ Christophe Nguyen, ${ }^{\ddagger}$ Mathieu Galibert, ${ }^{\dagger}$ Francisco Santos-Schneider ${ }^{\ddagger}$ Gudrun Aldrian, ${ }^{\dagger}$

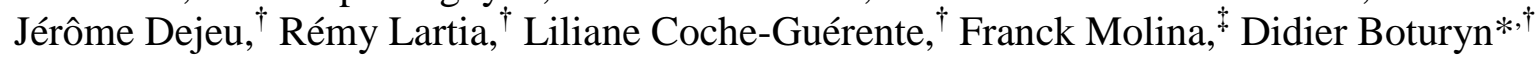 \\ †Univ. Grenoble-Alpes, CNRS, DCM UMR 5250, 570 rue de la chimie, CS 40700, 38058 Grenoble Cedex 9, France. \\ Email: didier.boturyn@univ-grenoble-alpes.fr \\ ${ }^{*}$ Sys2Diag, CNRS - ALCEDIAG, Cap delta/Parc Euromédecine, 1682 rue de la Valsière, CS 61003, 34184 Montpellier \\ Cedex 4, France.
}

\begin{abstract}
Antibodies play a major role in clinical diagnostics and biopharmaceutical analysis, but are also a class of drugs that are regularly used to treat numerous diseases. The identification of antibody-epitope binding sites is then of great interest to many emerging medical and bioanalytical applications, particularly to design mAb mimics taking advantage of amino acids residues involved in the binding. Among relevant antibodies, the monoclonal antibody rituximab has received a significant attention as it is exploited to treat several cancers including non-Hodgkin's lymphoma and chronic lymphocytic leukemia, as well as some autoimmune disorders such as rheumatoid arthritis. The binding of rituximab to the targeted cells occurs via the recognition of the CD20 epitope. A crystallography study has shown that the binding area, named paratope, is located at the surface of rituximab. Combining the SPOT method and the complementary surface plasmon resonance technique allowed us to detect an extended recognition domain buried in the pocket of the rituximab Fab formed by four $\beta$-sheets. More generally, the present study offers a comprehensive approach to identify antibody-epitope binding sites.
\end{abstract}

Biomolecular recognition is central to many biological processes governing cell fate. In this context, the monoclonal antibodies (mAbs) are able to bind to a specific antigen triggering the death of the targeted cell. ${ }^{1}$ To date, mAbs are increasingly used for treatment of a variety of diseases. ${ }^{2}$ In particular, rituximab (RTX), first FDA (Food and Drug Administration) approved therapeutic $\mathrm{mAb}$, is routinely used for the treatment of several types of lymphoma as well as some autoimmune disorders. ${ }^{3}$ RTX was shown to target a transmembrane protein named CD20 (cluster of differentiation 20), which is highly expressed on most healthy and malignant B cells but not on precursor B cells. ${ }^{4}$ The identification of CD20 recognized by Rituximab has raised great interest in recent years especially to better apprehend RTX mechanism and action. Additionally, understanding molecular recognition could pave the way for structure-based design of bioactive molecules. Very recently, it has been shown by using cryo-electron microscopy (cryoEM) that the native CD20 structure is a compact dimeric form allowing RTX cross-links that can induce complement recruitment. ${ }^{5,6}$ This result corroborates other studies indicating that CD20s are not expressed in monomeric form, but organized into supramolecular protein complexes. ${ }^{7,8}$ In parallel, our group has studied the effect of CD20 density on the recognition by RTX, which is known to be a critical point for the therapeutic response. ${ }^{9}$ An average critical inter-CD20 spacing of nearly $2 \mathrm{~nm}$ confers the best conditions for RTX binding. This value is in excellent agreement with cryo-EM structures. ${ }^{5,6}$ It is important to note that this finding suggests RTX contacts with two different epitopes. ${ }^{5}$ Regarding the identification of the antigen-binding site of the antibody, a pioneer crystal structure of the RTX antigen-binding fragment (Fab) in complex with its epitope, i.e. a protein fragment representing the large extracellular loop of the CD20 protein, was also reported. ${ }^{10}$ This study revealed that a unique short CD20 epitope interacts with the outer surface of RTX. The differences observed from cryo-EM structures highlight a recognition via an extended RTX-CD20 region.

Among approaches exploited for the characterization of the interaction between a mAb and its epitope, the SPOT method is typically used for antigenic epitope mapping. ${ }^{11-13}$ The strategy consists in a parallel peptide synthesis carried out simultaneously on cellulose membranes to generate large libraries of small antigen fragments that are subsequently used to reveal the antibody's epitope. The SPOT method was applied to map a broad area of interactions between endonucleases and oligonucleotides, ${ }^{14}$ peptides and metals, ${ }^{15}$ cytokines and their receptors, ${ }^{16}$ to evaluate substrate specificity of proteases, ${ }^{17}$ to develop therapeutic vaccines, ${ }^{18}$ but also to find antimicrobial peptides. ${ }^{19}$ Interestingly, this method can be used to identify amino acids and peptide fragments from the antibody paratope that contribute to the antigen binding. ${ }^{20}$ Following this strategy, in the present work we report the characterization of the binding site of RTX to CD20 by using the SPOT method and complementary surface plasmon resonance (SPR) experiments. The SPR technique, which consists in studying biomolecular interactions on surface, will be exploited to corroborate SPOT analysis, and to determine the antigen-binding site with high accuracy. Our results provide additive information to the crystallo- 
graphic data and cryo-EM studies, and above all, new insights on the interaction between RTX and its CD20 epitope with a paratope buried in a pocket formed by four $\beta$-sheets that can be exploited to design RTX mimics.

\section{EXPERIMENTAL SECTION}

All organic compounds were purchased from VWR Inter-national S.A.S. (Fontenay-sous-Bois, France), Sigma-Aldrich (SaintQuentin Fallavier, France), unless otherwise specified in the text. Rituximab (MabThera) was provided gracefully by Genentech, Inc. (San Francisco, US). Solution of infusion was provided at a concentration of $10 \mathrm{mg} / \mathrm{mL}$ (i.e. $69.5 \mu \mathrm{M}$ ).

SPOT synthesis and analysis. For CD20 binding site screening, SPOT synthesis technique was used to obtain immobilized peptides on cellulose membrane covering the RTX Fab sequence light $(\mathrm{L})$ and heavy $(\mathrm{H})$ chain variable region. We synthesized five copies of $105(\mathrm{~L})$ and $121(\mathrm{H})$ overlapping linear hexapeptides and two copies of $99(\mathrm{~L})$ and $115(\mathrm{H})$ overlapping linear dodecapeptides, frameshifted by one residue, covering the amino acid sequence of RTX Fab, as well as five copies of 105 (L) and $121(\mathrm{H})$ cyclic octapeptides and four series of the selected regions for alanine scanning in order to determine critical amino acids (for synthesis details see the Supplementary materials). Incubation of SPOT membranes with fluorescent Cyanine 5-containing sCD20 allowed identification of positive sequences.

Synthesis of sCD20 epitope and scrambled sCD20. The sCD20 (soluble CD20) peptide used in this work and reported X-ray studies ${ }^{10}$ corresponds to a 25 -amino-acid sequence that is located in the extracellular part of the full CD20 protein. The scrambled sCD20 peptide used as a reference was composed of the same amino-acids in a random order except cycteines 5 and 25 involved in disulfide bridge. The sCD20 and scrambled sCD20 sequences are respectively NIYNCEPANPSEKNSPSTQYCYSIQ and SATNCNSEYQNEPNYIPYSQCKPIS. Synthesis of the protected peptides was carried out using the Fmoc/t-Bu strategy on a peptide synthesizer (Syro2, Biotage) using 2-chlorotritylchloride ${ }^{\circledR}$ (See the Supplementary materials). Reaction with 4-pentynoic acid was performed on the resin to incorporate the alkyne function at the $\mathrm{N}$-terminus. After cleavage and removal of protecting groups, disulfide bridge formation of peptides $(500 \mu \mathrm{M})$ was performed under mild oxidative conditions $(0.1 \mathrm{M}$ TrisBase, 5\% DMSO, 20 $\mathrm{mM}$ guanidine) during 24 hours. Peptides were then purified by RP-HPLC and their concentration quantified by a UV spectrophotometer.

Synthesis of RTX peptide fragments. All peptides were prepared by using the same procedure than sCD20. Their concentrations were quantified by a UV spectrophotometer according to the peptide sequences.

SPR analyses. SPR analyses were carried out on a Biacore T200 (GE Healthcare). Gold sensor chips were cleaned by UV-ozone treatment (Jelight, Irvine, CA, USA) during 10 min after being rinsed with ultrapure water and ethanol. The cleaned gold surfaces were then functionalized according to the following procedure. ${ }^{9}$ Mixed self-assembled monolayers (SAMs) were formed at room temperature by immersing gold sensors overnight in a thiol mixture of $12.5 \% \mathrm{HS}-\left(\mathrm{CH}_{2}\right)_{11}-\mathrm{EG}_{6}-\mathrm{N}_{3}$ and $87.5 \% \mathrm{HS}_{-}\left(\mathrm{CH}_{2}\right)_{11}-\mathrm{EG}_{4}-\mathrm{OH}$ $(1 \mathrm{mM}$ total thiol concentration in EtOH). Sensor chips were then rinsed with ethanol and blow-dried with nitrogen gas. The surfaces were inserted in the Biacore T200 device. For the grafting of sCD20 antigen, manual runs were conducted with milli-Q water as running solvent at $2 \mu \mathrm{L} / \mathrm{min}$. Azide-alkyne cycloaddition permitted the covalent grafting of scrambled sCD20 on channel 1 as reference, and sCD20 on channel 2. The reagent mixture is composed of sCD20 alkyne/ scrambled sCD20-alkyne $(200 \mu \mathrm{M}), \mathrm{CuSO} 4$ $(500 \mu \mathrm{M})$, Tris[(1-benzyl-1H-1,2,3-triazol-4-yl)methyl]amine (TBTA) (500 $\mu \mathrm{M})$, and (+)-sodium L-ascorbate (3 mM), in DMSO: $\mathrm{H}_{2} \mathrm{O}(1: 1)$. As the microfluidic system is sensitive to high concentrations of DMSO, time of injection was controlled and optimized at $8 \mathrm{~min}$ for each channel, with $8 \mathrm{~min}$ wait between each injection. The large change of solution refractive index during the injection prevents monitoring of the grafting process in real-time. However, the quantity of immobilized antigen could be evaluated by comparing the shifts in resonance units before and after the injection. ${ }^{9}$ For the peptide fragment analyses, the running buffer $(\mathrm{RB})$ consisted of PBS $(1 \times)$, citrate $(3 \mathrm{mM})$ and polysorbate P80 $(0.005 \%)$. Flow cell 1 was functionalized with scrambled sCD20 to provide a reference surface. Binding assays were conducted using a flow rate of $30 \mu \mathrm{L} / \mathrm{min}$ at $25^{\circ} \mathrm{C}$ on different surfaces displaying 51.5 pmol. $\mathrm{cm}^{-2}$ of sCD20 peptide in average. Fab fragments were dissolved in RB and injected using the high performance Biacore method (injection time: $200 \mathrm{~s}$, stabilization time: $600 \mathrm{~s}$ ) at different concentrations: 0, 25, 50, 90, 180, 300, 500, 800 $\mu \mathrm{M}$. Curves obtained at $25 \mu \mathrm{M}$ and generally flat are not presented here. A regeneration step was necessary between each injection, and was performed by injecting a solution of Glycine- $\mathrm{HCl}(10 \mathrm{mM}, \mathrm{pH} 2)$ for $10 \mathrm{~s}$. The stability of the sCD20-functionalized surfaces was checked by achieving regular RTX injections at $1 \mu \mathrm{M}, 100 \mathrm{nM}$ and $10 \mathrm{nM}$ at different times of the binding assays. Because the functionalized surfaces are stable, several peptides were sequentially screened on the same sCD20-functinalized sensor. For duplicates, the order of injection of the peptides was always modified to be sure that results were not caused by sCD20 film degradation. Some peptides were also screened on several surfaces to check reproducibility of observed curve magnitudes. Because of a lack of solubility, peptides B4-1 and B4-2 synthesized for alanine scanning analysis were tested in RB + DMSO 2\%. The peptide from which they are derivated (B4) was injected in both buffers with and without DMSO to ensure that DMSO did not have any impact on recognition properties. The presented binding sensorgrams correspond to double-referenced sensorgrams using reference flow cell 1 and preceding buffer blank subtractions to remove the non-specific adsorption of RTX and the change in refractive index respectively. The SPR kinetic evaluation software of Biacore T200 (evaluation Software 2.0.1) was used to fit the data with a steady-state model, which demonstrated that affinities of isolated RTX fragments are too low to be calculated with accuracy in the examined range of concentrations. 


\section{RTX VL domain}

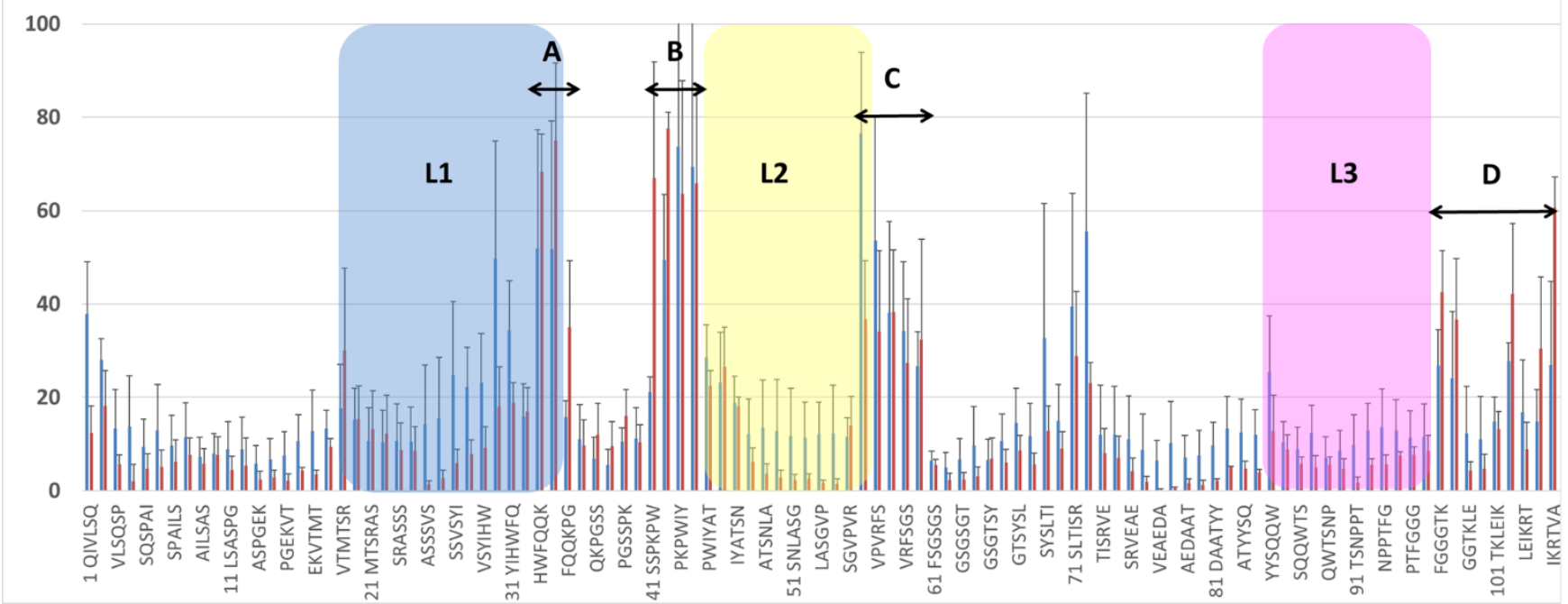

\section{RTX VH domain}

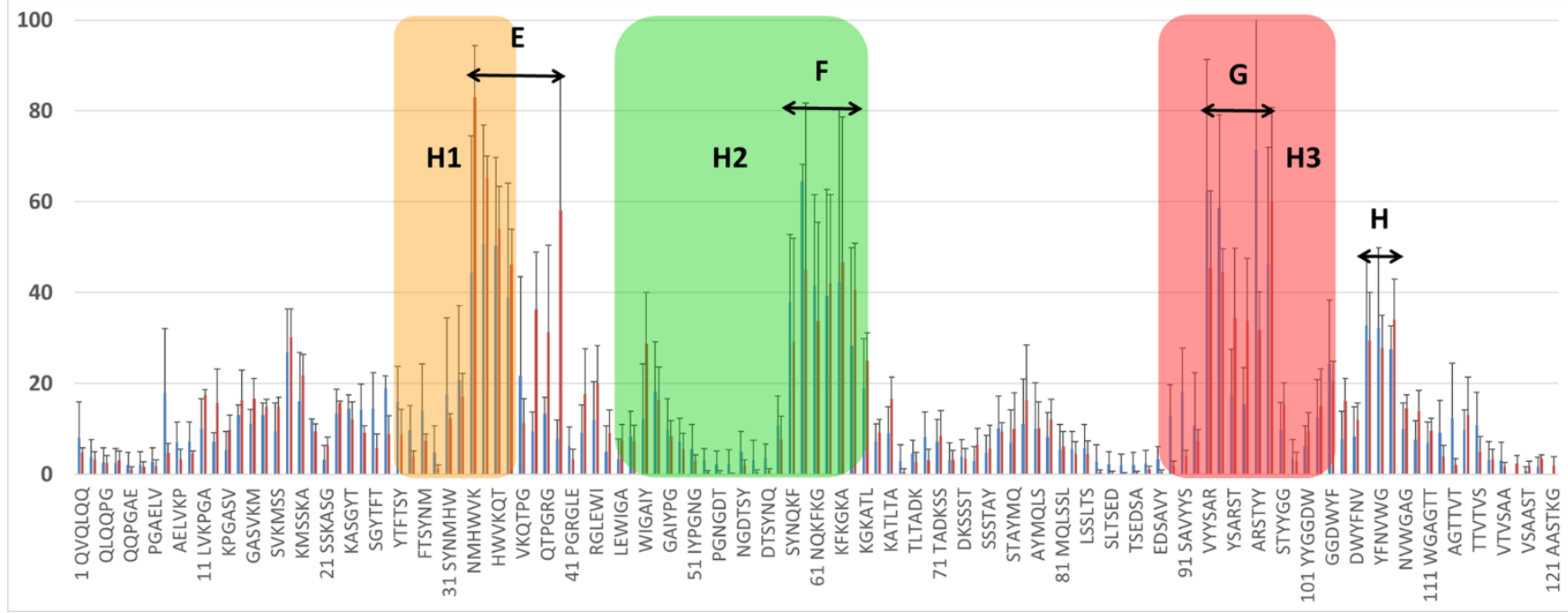

Figure 1. Reactivity pattern of sCD20 with overlapping hexapeptides (blue bars) and their cyclic analogues (red bars) from the VH (variable region of the heavy chain) and VL (variable region of the light chain) amino acid sequences of RTX. Peptides were prepared by the SPOT method and probed for reactivity with Cy5-containing sCD20. Fluorescence intensity is shown in arbitrary units. Strong binding was indicated by a double-headed arrow (RTX sequences A to H). The numbering and the CDR location (L1, L2, L3, H1, H2 and H3) are according to Kabat. ${ }^{23}$ To avoid undesired cyclization, cysteine residues within the Fab were substituted with serine. The amino acid sequence of the cyclic peptides

\section{RESULTS AND DISCUSSION}

To map the discontinuous binding site of RTX that recognizes the CD20 epitope, we prepared a set of 226 overlapping linear hexapeptides and dodecapeptides (See the sequences in the Supporting Information), which represents the full sequence of the RTX variable domain (VH and VL, respectively for the heavy and the light chain), by using the SPOT-synthesis method. ${ }^{11,12,20}$ As linear peptides contain many conformers decreasing generally their biological activity, ${ }^{21}$ we decided to carry out an additional SPOTsynthesis of cyclopeptides comprising sequences corresponding to the linear peptides. For this purpose, two additional cysteine

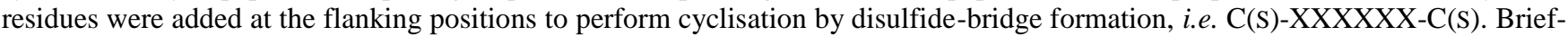
ly, linear peptides were synthesized on cellulose membranes by using Fmoc-based solid phase peptide synthesis (SPPS), and cyclisation was performed under mild oxidative conditions. In parallel, we synthesized a soluble part of the CD20 protein (sCD20) corresponding to the large extracellular loop of the CD20 protein (amino acids $\mathrm{N}^{163}$ to $\mathrm{Q}^{187}$ ) which is known to contain the binding epitope of RTX. ${ }^{10}$ Other CD20 protein regions, such as a small extracellular loop (seven amino acids) and hydrophobic membranespanning domains, are non-immunogenic to RTX. It is important to note that the cyclic conformation of sCD20 is constrained by a disulfide bond. ${ }^{10}$ We also prepared a scrambled peptide of $\mathrm{sCD} 20$ used as a negative control. Introduction of a cyanine-5 dye at the $\mathrm{N}$-terminus of sCD20 and scrambled sCD20 was carried out to evaluate the epitope mapping.

Membranes containing around $50 \mathrm{nmol}$ of peptides per spot (4 $\mathrm{mm}$ in diameter) were then incubated with cyanine-5 labeled sCD20 at concentration of $20 \mathrm{nM}$. Figure 1 shows the average binding pattern of sCD20 on the different spots functionalized with 
linear and cyclic peptides. The overall observation is that numerous peptides from RTX sequence are able to bind the sCD20 but not the scrambled sCD20 (Figure S1), indicating the selectivity of the interaction. As expected, strong fluorescence signals were observed within the hypervariable loops from the heavy chain of RTX namely the complementarity-determining regions (CDRs) $\mathrm{H} 1, \mathrm{H} 2$ and $\mathrm{H} 3$, respectively the sequences from regions E, F and $\mathrm{G}$. This is in agreement with the general observation for the hypervariable loops from the heavy chain that are the main contributors for antigen recognition. ${ }^{22}$ We also detected a strong sCD20 binding with the CDR L1 (sequences A) and the neighboring regions of the CDR L2 (sequences B and C) that belong to the RTX light chain. Today, it is recognized that not all CDRs are involved in the traditionally defined CDRs and that other mAb residues outside the CDRs can contribute to the antigen binding. ${ }^{22}$ Two other regions (sequences D and $\mathrm{H}$ ) show moderate binding. A similar binding pattern was observed for the linear dodecapeptides (Figure S2). It is important to note that reactivity of linear and cyclic peptides is roughly the same. Interestingly, SPOT analysis shows that the binding site of CD20 is located in a small pocket formed by $\beta$-sheets that belong to mAb hypervariable loops (Figure 2), in particular the sequences A, B, E, G and $\mathrm{H}$ that correspond respectively to CDRs L1, L2, H1 and H3.

Comparison with the binding site obtained from the crystal structure ${ }^{10}$ reveals differences in the location of the sCD20. While the RX structure shows that the antigen is positioned at the surface of the mAb, SPOT analysis shows that sCD20 is deeply embedded into the RTX Fab (Figure 2). From the crystallography study, the CDR H3 interacts with the CD20 via the peptide fragment ${ }^{99}$ STYYGGDW ${ }^{106}$, while using the SPOT method recognition occurs with other flanking amino acids within the fragments ${ }^{93}$ VYYSARSTYYG ${ }^{103}$ and ${ }^{106}$ WYFNVWGA $^{113}$. Similar observation is shown for $\mathrm{H} 1$ as we found an extended recognition area ${ }^{33}$ NMHWVKQTPGRGL ${ }^{45}$ from SPOT vs. ${ }^{33} \mathrm{NMH}^{35}$ from crystallography study. Strong difference was also obtained for the CDR $\mathrm{H} 2$ : a deep interaction was found using SPOT $\left({ }^{59} \mathrm{SYNQKFKGKA}^{68}\right)$ in comparison to sequence obtained from the crystal structure $\left({ }^{47}\right.$ WIGAIYPGNGDTS $\left.{ }^{59}\right)$. Concerning the light chain, the differences are even more pronounced. From the crystal structure, the recognition area is only composed of the peptide fragment ${ }^{90}{ }^{W T S N P P}{ }^{95}$ (L3). The SPOT method reveals fragments deeper in the Fab close to CDRs L1 and L2 such as ${ }^{33} \mathrm{HWFQQKPG}^{40},{ }^{41} \mathrm{SSPKPWIYA}^{49}$ and ${ }^{56} \mathrm{GVPVRFSGSG}^{65}$ (Figure 2).

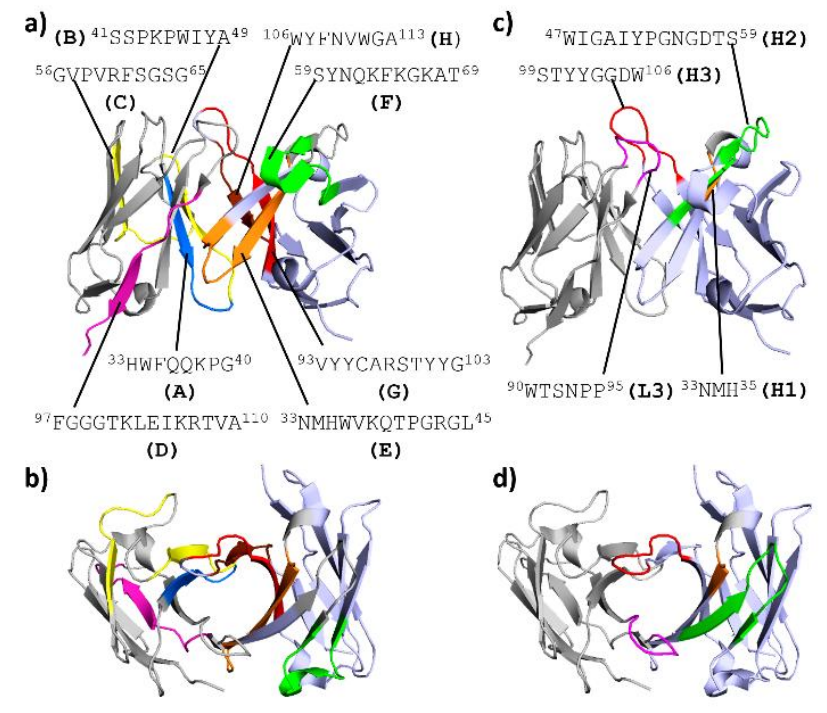

Figure 2. RTX recognition areas obtained from the SPOT method with (a) side-view and (b) top-view, and from X-ray diffraction ${ }^{10}$ with (c) side-view and (d) top-view. Sequences A-D from the VH of RTX and sequences E-H from the VL of RTX are represented.

Table 1. Determination of critical residues from RTX Fab involved in RTX-CD20 recognition. ${ }^{\mathrm{a}}$

\begin{tabular}{|c|c|c|c|}
\hline CDR loops & Regions & Sequences used for Ala-scan study & Critical amino acids \\
\hline L1 & A & ${ }^{33} \mathrm{HWFQQKP}^{39}$ & $\mathrm{~W}^{34}, \mathrm{~F}^{35}, \mathrm{~K}^{38}$ \\
\hline $\mathrm{L} 2$ & $\mathrm{~B}^{\mathrm{b}}$ & ${ }^{42}$ SPKPWIYA $^{49}$ & $\mathrm{~K}^{44}, \mathrm{~W}^{46}, \mathrm{Y}^{48}$ \\
\hline L2 & $\mathrm{C}^{\mathrm{b}}$ & ${ }^{56}$ GVPVRFSGS $^{64}$ & $\mathrm{R}^{60}, \mathrm{~F}^{61}$ \\
\hline L3 & $\mathrm{D}^{\mathrm{b}}$ & ${ }^{97}$ FGGGTKLEIKRTVA ${ }^{110}$ & ND \\
\hline H1 & $\mathrm{E}$ & ${ }^{31}$ SYNMHWVKQTP $^{41}$ & $\mathrm{~W}^{36}, \mathrm{~K}^{38}$ \\
\hline $\mathrm{H} 2$ & $\mathrm{~F}$ & ${ }^{59} \mathrm{SYNQKFKGKA}^{68}$ & - \\
\hline $\mathrm{H} 3$ & $\mathrm{G}$ & ${ }^{93}$ VYYSARSTYYG ${ }^{103 c}$ & $\mathrm{Y}^{94}, \mathrm{Y}^{95}, \mathrm{R}^{98}, \mathrm{Y}^{101}, \mathrm{Y}^{102}$ \\
\hline $\mathrm{H} 3$ & $\mathrm{H}^{\mathrm{b}}$ & ${ }^{106}$ WYFNVWGA $^{113}$ & $\mathrm{~W}^{106}, \mathrm{Y}^{107}, \mathrm{~F}^{108}, \mathrm{~W}^{111}$ \\
\hline
\end{tabular}

(a) Cyclic octapeptide-containing SPOT membrane was prepared by using the different peptide sequences and characterized as described. ${ }^{11,12,20}$ Two additional cysteine residues were added at the flanking positions to perform cyclisation by disulfide-bridge formation. (b) Sequences close to CDRs. (c) Cysteine residue was substituted with serine. ND: not determined. 
In order to identify amino acid residues that contribute in the binding with sCD20, a systematic alanine-scanning was carried out on the positive sequences (Table 1). From the SPOT reactivity pattern (Figures S4 and S5), 20 amino acids were shown to be critical for the CD20 recognition (Table 1). Most of them belong to $\beta$-sheets from CDRs H1, H3, L1 and L3 that form a wide pocket (regions $\mathrm{A}, \mathrm{B}, \mathrm{E}$ and $\mathrm{G}$ in the figure $2 \mathrm{~b}$ ). Among these residues, only $\mathrm{Y}^{102}$ and $\mathrm{W}^{106}$ from the CDR $\mathrm{H} 3$ were shown to be involved in the CD20 recognition on the crystal structure. ${ }^{10}$ Taken together these data tend to confirm that we found a CD20 binding site deeper in the RTX Fab than the one obtained from the crystallography study.

To further characterize and validate the functional relevance of the positive peptides highlighted by the SPOT method, SPR experiments were performed. Very recently, we have designed self-assembled monolayer (SAMs) on gold surfaces containing adjusted SCD20 mass densities for an optimal RTX recognition with an average $\mathrm{K}_{\mathrm{D}}$ value (32 nM) close to values previously reported from biological analysis with B cells (apparent $\mathrm{K}_{\mathrm{D}}$ between 5 and $20 \mathrm{nM}$ ). ${ }^{9}$ These SAMs are formed by incubating gold sensors in a thiol mixture of varying percentages of $\mathrm{HS}-\left(\mathrm{CH}_{2}\right)_{11}-\mathrm{EG}_{6}-\mathrm{N}_{3}$ and $\mathrm{HS}-\left(\mathrm{CH}_{2}\right)_{11}-\mathrm{EG} 4-\mathrm{OH}$, then copper-catalyzed azide/alkyne cycloaddition permits the covalent grafting of alkyne-CD20. The best conditions for RTX binding were found for an average critical inter-CD20 spacing of $2 \mathrm{~nm}\left(46.1 \pm 10.5\right.$ pmol.cm $\left.{ }^{-2}\right)$ using a ratio of $12.5 \% \mathrm{HS}-\left(\mathrm{CH}_{2}\right)_{11}-\mathrm{EG}_{6}-\mathrm{N}_{3}$. We thus prepared surfaces displaying sCD20 in adequate mass density $\left(51.5 \mathrm{pmol} . \mathrm{cm}^{-2}\right)$ but also for negative control displaying scrambled sCD20. In parallel, 25 cyclopeptides from the positive regions were prepared by SPPS as a soluble form (Table S1). An additional serine at the Nterminal was added to improve the solubility of the cyclic peptides. SPR measurements performed with non-labeled and purified peptides give rise to refined results, as the chosen concentration range of injected peptides delimits a specific $K_{D}$ section where the interaction is observable, and suppresses weaker affinities. Binding assays were then done by injecting the cyclic peptides (concentration range from 50 to $800 \mu \mathrm{M}$ ) over the sCD20-surfaces as described. ${ }^{9}$ To ensure over time the good recognition properties of the surfaces, rituximab injections were performed and consistency of the signal intensities was verified (Figure S6). The screening results show that four peptides (sequences A1, B4, E2 and G1) have a significant binding to sCD20-coated surfaces (Figure 3) while negligible binding was observed for peptides from regions C and F in the concentration range studied (Figures S8-S12). Peptides from region $\mathrm{H}$ were found to be insoluble under the analysis conditions. It is worth pointing out that A1, B4, E2 and G1 sequences are related to the most reactive regions of RTX. Interestingly, the positive peptides belong to the $\beta$-sheets forming the edge of the pocket within the core of the Fab and confirm the SPOT analyses.

Calculated from data fittings, $\mathrm{K}_{\mathrm{D}}$ related to the interaction between the sCD20 and RTX fragments are in the millimolar order, which is beyond the maximum concentration that could be injected $(800 \mu \mathrm{M})$. As a consequence, it was not possible to provide accurate $\mathrm{K}_{\mathrm{D}}$ values. Considering that the RTX binding site is discontinuous, this millimolar $\mathrm{K}_{\mathrm{D}}$ range was expected.

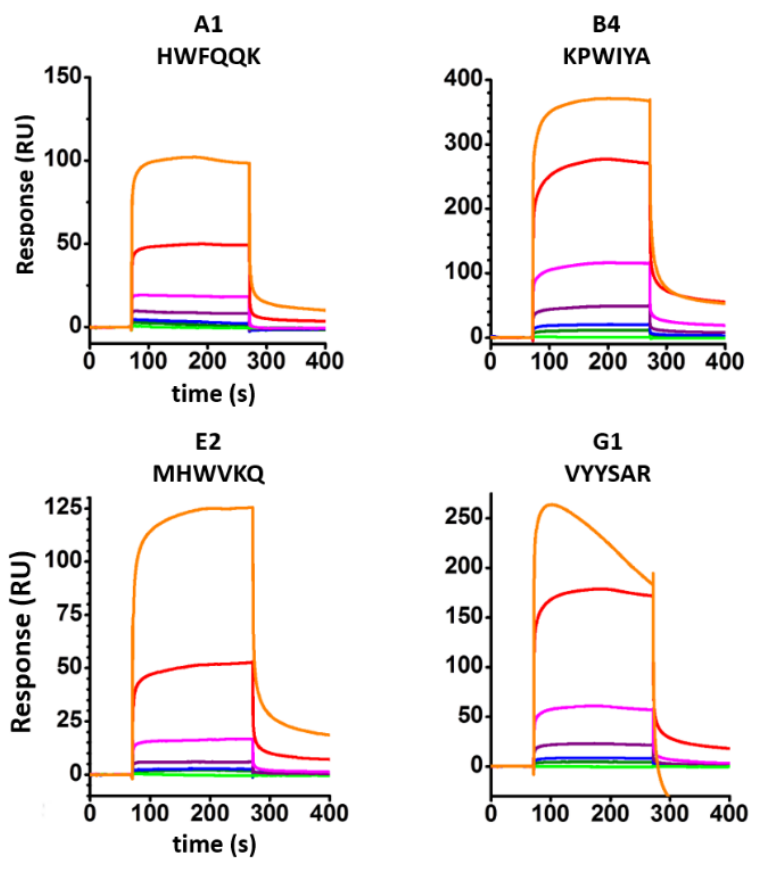

Figure 3. SPR sensorgrams of cyclopeptides A1, B4, E2 and G1. The amino acid sequence of the cyclic peptides is S-C(S)-XXXXXX$\mathrm{C}(\mathrm{S})$. The concentration range presented here is $0,50,90,180,300,500,800 \mu \mathrm{M}$. The solutions were injected on $\mathrm{CD} 20$-functionalized surfaces $\left(51.5\right.$ pmol.cm $\left.{ }^{-2}\right)$. The slight curve decrease observed when injecting G1 at $800 \mu \mathrm{M}$ is due to a loss of solubility of the peptide at high concentration.

We similarly identified by SPR critical amino acids by performing the alanine scanning of the peptides A1, B4, E2 and G1 (Figure 4, Figures S13-S16). Substitution of $\mathrm{W}^{34}, \mathrm{~F}^{35}, \mathrm{~K}^{44}, \mathrm{~W}^{46}$, and $\mathrm{Y}^{48}$ from the light chain and $\mathrm{W}^{36}, \mathrm{~K}^{38}, \mathrm{Y}^{94}, \mathrm{Y}^{95}$, and $\mathrm{R}^{98}$ from the heavy chain results in a total loss of affinity for SCD20 as found in SPOT experiment, suggesting that their presence is essential for antigen recognition. Additional residues were shown to be important in the binding site such as $\mathrm{I}^{47}$ (Light chain), $\mathrm{M}^{34}$ and $\mathrm{V}^{93}$ (Heavy chain). It is worth noting that no critical amino acids were found in the regions $\mathrm{C}$ and $\mathrm{F}$, which are outside the pocket delim- 
ited by the $\beta$-sheets forming the pocket (Figures $2 \mathrm{a}$ ). Surprisingly, the substitution of a few amino acids to alanine $\left(\mathrm{H}^{33}, \mathrm{Q}^{36}\right.$ and $\mathrm{Q}^{37}$ within the region $\mathrm{A}, \mathrm{Q}^{39}$ within the region $\mathrm{E}$ ) has improved the $\mathrm{sCD} 20$ binding. Interestingly, the results obtained by the SPOT method and the SPR are extremely similar (table 2), even though experimental conditions of both techniques are different, as sCD20 was used in solution (SPOT analyses) and on surface (SPR experiments).

Table 2. Determination of critical residues from RTX Fab involved in RTX-CD20 recognition by SPOT and SPR experiments.

\begin{tabular}{|c|c|c|c|}
\hline CDR loops & Regions & Critical residues determined by SPOT method & Critical residues determined by SPR \\
\hline L1 & $\mathrm{A}$ & $\mathrm{W}^{34}, \mathrm{~F}^{35}, \mathrm{~K}^{38}$ & $\mathrm{~W}^{34}, \mathrm{~F}^{35}$ \\
\hline L2 & $\mathrm{B}$ & $\mathrm{K}^{44}, \mathrm{~W}^{46}, \mathrm{Y}^{48}$ & $\mathrm{~K}^{44}, \mathrm{~W}^{46}, \mathrm{I}^{47}, \mathrm{Y}^{48}$ \\
\hline $\mathrm{H} 1$ & $\mathrm{E}$ & $\mathrm{W}^{36}, \mathrm{~K}^{38}$ & $\mathrm{M}^{34}, \mathrm{~W}^{36}, \mathrm{~K}^{38}$ \\
\hline $\mathrm{H} 3$ & $\mathrm{G}$ & $\mathrm{Y}^{94}, \mathrm{Y}^{95}, \mathrm{R}^{98}, \mathrm{Y}^{101}, \mathrm{Y}^{102}$ & $\mathrm{~V}^{93}, \mathrm{Y}^{94}, \mathrm{Y}^{95}, \mathrm{R}^{98}$ \\
\hline
\end{tabular}

A

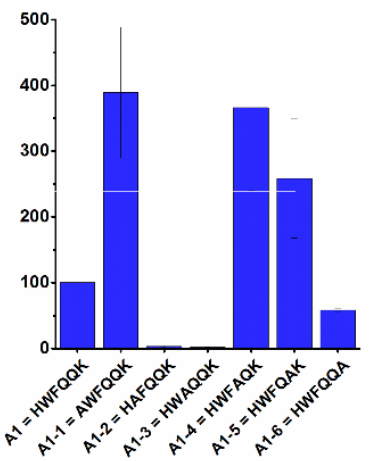

C

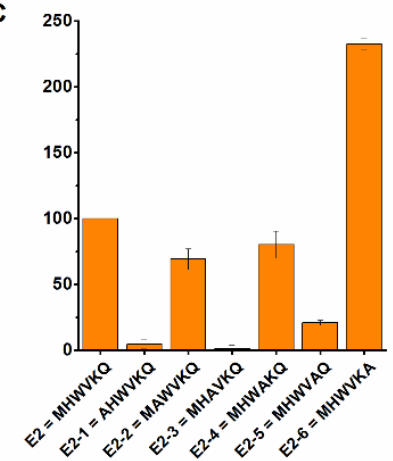

B
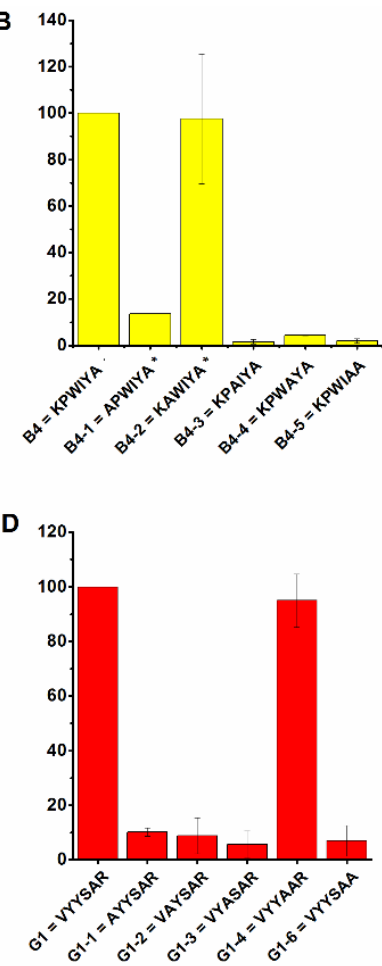

Figure 4. SPR experiments of alanine scanning of A) peptides from A1, B) peptides from B4, C) peptides from E2 and D) peptides from

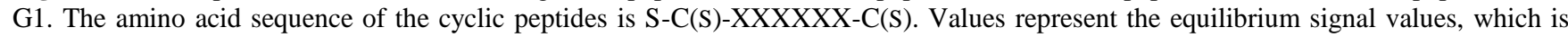
normalized to the original sequence (100\%). * Sequences tested in 2\% DMSO.

The soluble peptides selected from SPOT analysis that display efficient CD20 recognition on surface enable to refine the binding site of RTX to the CD20 epitope. As shown in figure 5a, critical amino acid residues are very close to each other. They are located in both heavy (CDRs H1 and H3) and light chains (CDRs L1 and L2) in an open pocket. Conversely, X-ray structure analysis suggests that the interaction occurs at the surface on the mAb with critical residues belonging to external loops of CDRs L3, H1, H2 and H3 (Figure 5b). Our results indicate that RTX Fab contains an extended paratope region. Altogether, we propose a binding of CD20 to the surface of the RTX via the recognition of the H1, H2, H3 and L3 loops as depicted in figure 5b, then a slip of the epitope between the four $\beta$-sheets formed by H1, H3, L1 and L2. To date, there is strong evidence that the flexibility of H3 loop has a huge impact on the antigen binding process, ${ }^{22,24,25}$ and may contribute to the antigen drift toward the Fab pocket. This highlights a dynamic binding mechanism. It is important to note that this dynamic mechanism is impossible to characterize using X-ray analysis as this technique identifies only one of the most stable interaction. 
a)
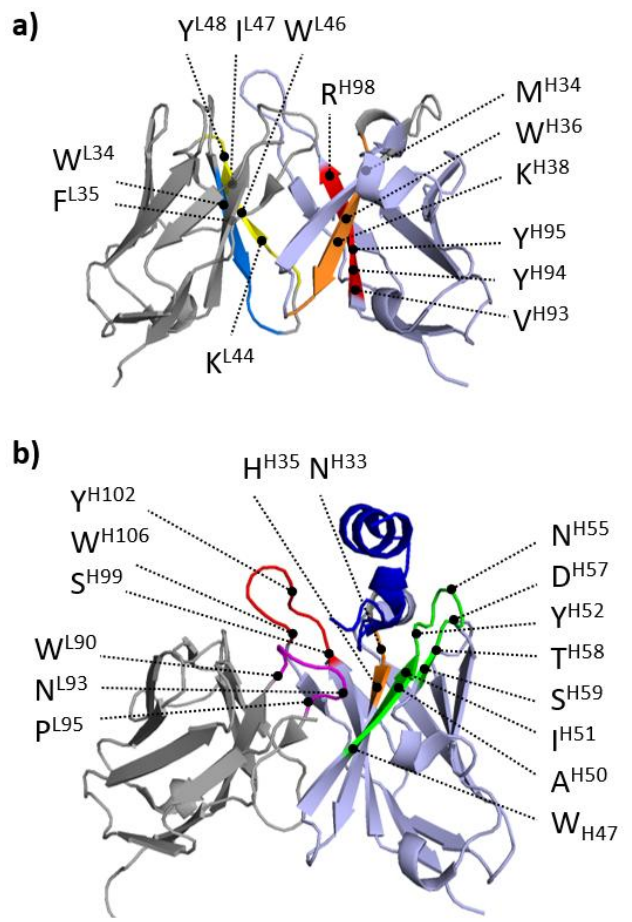

Figure 5. Comparison of RTX recognition binding site obtained from (a) the combined SPOT-SPR method and (b) X-ray diffraction. ${ }^{10}$ The CD20 epitope is shown in dark blue. Critical amino acids are represented; $\mathrm{L}=$ light chain, $\mathrm{H}=$ heavy chain.

Furthermore, the results presented here should facilitate the rational design of RTX mimics. As mAbs have several limitations related to their nature (expensive biotechnological procedure, large size which limits tissue penetration), the design of peptidomimetics that focuses on small loops represents a novel strategy to produce new therapeutic compounds for the treatment of several malignancies and other diseases. In this context, our group is developing new RTX mimics and results will be reported in due time.

\section{CONCLUSION}

In recent years, much research effort has been devoted to determine the antibody-epitope binding site as the range of therapeutic mAbs has been remarkably increasing since the beginning of the 21 st century. While the commonly accepted view has been that Xray crystallography remains the key technique with NMR to identify the site of a protein-protein interaction, our results suggest that the dual SPOT-SPR methodology is also appropriate to identify critical residues involved in the recognition domain. Using the dual SPOT-SPR methods, we have mapped the rituximab domain that recognizes the CD20 epitope. Interestingly, the binding site differs from that obtained by X-ray structure analysis opening questions regarding the use of supplementary methods in addition to crystallography to determine the mAb binding site. Together, our results provide new insights in CD20-RTX recognition and could pave the way for the discovery of mAb mimics by means of amino acids residues involved in the binding.

\section{ASSOCIATED CONTENT}

\section{Supporting Information}

Detailed description on SPOT method, SPOT membranes, preparation of CD20 antigen peptide, preparation of Fab fragments, surface stability, and additional SPR sensorgrams were available in the supporting information. The Supporting Information is available free of charge on the ACS Publications website.

\section{AUTHOR INFORMATION}

\section{Corresponding Author}

*E-mail: didier.boturyn@univ-grenoble-alpes.fr.

\section{Present Addresses}

$\dagger$ If an author's address is different than the one given in the affiliation line, this information may be included here.

\section{Author Contributions}


The manuscript was written through contributions of all authors. All authors have given approval to the final version of the manuscript.

\section{ACKNOWLEDGMENT}

This work was supported by the CNRS, the University Grenoble Alpes, the "Communauté d'agglomération Grenoble-Alpes Métropole" (Nanobio program), and by the "Agence Nationale de la Recherche" including "Mimobody" research support (ANR13-BS07-0014-01), LabEx ARCANE and CBH-EUR-GS (ANR-17-EURE-0003). We acknowledge the support from the ICMG chemistry platform, Grenoble, on which the peptide synthesis has been performed, the ICMG PCI platform, Grenoble, on which analyses were done, and Genentech Inc. (San Francisco), a member of Roche group, for providing Rituximab.

\section{REFERENCES}

(1) Strome, S. E.; Sausville, E. A.; Mann D. A mechanistic perspective of monoclonal antibodies in cancer therapy beyond targetrelated effects. Oncologist 2007, 12, 1084-1095.

(2) Ecker, D. M.; Dana Jones, S.; Levine, H. L. The therapeutic monoclonal antibody market. mAbs 2015, 7, 9-14.

(3) Klein, C.; Lammens, A.; Schäfer, W.; Georges, G.; Schwaiger, M.; Mössner, E.; Hopfner, K. P.; Umaña, P.; Niederfellner, G. Epitope interactions of monoclonal antibodies targeting CD20 and their relationship to functional properties. $m A b s$ 2013, 5, 2233.

(4) Casan, J. M. L.; Wong, J.; Northcott, M. J.; Opat, S. Anti-CD20 monoclonal antibodies: reviewing a revolution. Human Vaccines Immunother. 2018, 14, 2820-2841.

(5) Rougé, L.; Chiang, N.; Steffek, M.; Kugel, C.; Croll, T. I.; Tam, C.; Estevez, A.; Arthur, C. P.; Koth, C. M.; Ciferri, C.; Kraft, E.; Payandeh, J.; Nakamura, G.; Koerber, J. T.; Rohou, A. Structure of CD20 in complex with the therapeutic monoclonal antibody rituximab. Science 2020, 367, 1224-1230.

(6) Kumar, A.; Planchais, C.; Fronzes, R.; Mouquet, H.; Reyes, N. Binding mechanisms of therapeutic antibodies to human CD20. Science 2020, 369, 793-799.

(7) Cartron, G.; Watier, H.; Golay, J.; Solal-Celigny, P. From the bench to the bedside: ways to improve rituximab efficacy. Blood 2004, 104, 2635-2642.

(8) Li, M.; Xiao, X.; Zhang, W.; Liu, L.; Xi, N.; Wang, Y. Nanoscale distribution of CD20 on B- cell lymphoma tumour cells and its potential role in the clinical efficacy of rituximab. J. Microsc. 2014, 254, 19-30.

(9) Bar, L.; Dejeu, J.; Bano, F.; Lartia, R.; Richter, R. P.; Coche-Guérente, L.; Boturyn, D. Impact of antigen density on recognition by monoclonal antibodies. Anal. Chem. 2020, 92, 5396-5403.

(10) Du, J.; Wang, H.; Zhong, C.; Peng, B.; Zhang, M.; Li, B.; Huo, S.; Guo, Y.; Ding, J. Structural basis for recognition of CD20 by therapeutic antibody rituximab. J. Biol. Chem. 2007, 282, 15073-15080.

(11) Franck, R. Spot-synthesis: an easy technique for the positionally addressable, parallel chemical synthesis on a membrane support. Tetrahedron Lett. 1992, 48, 9217-9232.

(12) Franck, R. The SPOT-synthesis technique. Synthetic peptide arrays on membrane supports--principles and applications. $J$. Immunol. Methods 2002, 267, 13-26.

(13) Kramer, A.; Keitel, T.; Winkler, K.; Stöcklein, W.; Höhne, W.; Schneider-Mergener, J. Molecular basis for the binding promiscuity of an anti-p24 (HIV-1) monoclonal antibody. Cell 1997, 91, 799-809.

(14) Reuter, M.; Schneider-Mergener, J.; Kupper, D.; Meisel, A.; Mackeldanz, P.; Kruger, D. H.; Schroeder, C. Regions of endonuclease EcoRII involved in DNA target recognition identified by membrane-bound peptide repertoires. J. Biol. Chem. 1999, 274, 5213-5221.

(15) Malin, R.; Steinbrecher, A.; Semmler, W.; Noll, B.; Johannsen, B.; Frömmel, C.; Höhne, W.; Schneider-Mergener, J. Identification of technetium-99m binding peptides using combinatorial cellulose-bound peptide libraries. J. Am. Chem. Soc. 1995, 117, 11821-11822.

(16) Reineke, U.; Sabat, R.; Volk, H. D.; Schneider-Mergener, J. Mapping of the interleukin-10/interleukin-10 receptor combining site, Protein Sci. 1998, 7, 951-960.

(17) Laurent, N.; Haddoub, R.; Voglmeir, J.; Wong, S. C. C.; Gaskell, S. J.; Flitsch, S. L.; SPOT Synthesis of Peptide Arrays on Self- Assembled Monolayers and their Evaluation as Enzyme Substrates. ChemBioChem 2008, 9, 2592-2596.

(18) Duarte, C. G .; Alvarenga, L. M. ; Dias-Lopes, C.; Machado-de-Ávila, R. A.; Nguyen, C.; Molina, F.; Granier, C.; ChávezOlórtegui, C. In vivo protection against Tityus serrulatus scorpion venom by antibodies raised against a discontinuous synthetic epitope. Vaccine 2010, 28, 1168-1176.

(19) López-Pérez, P. M.; Grimsey, E.; Bourne, L.; Mikut, R.; Hilpert, K. Screening and optimizing antimicrobial peptides by using SPOT-synthesis. Front. Chem. 2017, 5:25.

(20) Laune, D.; Molina, F.; Ferrières, G.; Villard, S.; Bès, C.; Rieunier, F.; Chardès, T.; Granier, C. Application of the Spot method to the identification of peptides and amino acids from the antibody paratope that contribute to antigen binding. J. Immunol. Methods 2002, 267, 53-70.

(21) Kessler, H. Conformation and Biological Activity of Cyclic Peptides. Angew. Chem. Int. Ed. Engl. 1982, 21, $512-523$.

(22) Sela-Culang, I.; Kunik, V.; Ofran, Y. The structural basis of antibody-antigen recognition. Front. Immunol. 2013, 4:302. 
(23) Kabat, E. A.; Wu, T. T.; Bilofsky, H. Unusual distributions of amino acids in complementarity-determining (hypervariable) segments of heavy and light chains of immunoglobulins and their possible roles in specificity of antibody-combining sites. $J$. Biol. Chem. 1977, 252, 6609-6616.

(24) Tsuchiya, Y.; Mizuguchi, K. The diversity of H3 loops determines the antigen- binding tendencies of antibody CDR loops. Protein Sci. 2016, 25, 815-825.

(25) Fernández-Quintero, M. L.; Kraml, J.; Georges, G.; Liedl, K. R. CDR-H3 loop ensemble in solution - conformational selection upon antibody binding. mAbs 2019, 11, 1077-1088. 